\title{
À quelles conditions les salariés adhèrent-ils à un projet de crèche?
}

Private-professional life conciliation. Under what conditions will employees adhere to a project of a day-care facility?

Yvan Barel et Sandrine Frémeaux

\section{CpenEdition}

\section{Journals}

Édition électronique

URL : http://journals.openedition.org/travailemploi/4144

DOI : 10.4000/travailemploi.4144

ISSN : 1775-416X

Éditeur

DARES - Ministère du Travail

Édition imprimée

Date de publication : 30 mars 2009

Pagination : 81-89

ISSN : 0224-4365

\section{Référence électronique}

Yvan Barel et Sandrine Frémeaux, "À quelles conditions les salariés adhèrent-ils à un projet de crèche? », Travail et Emploi [En ligne], 117 | janvier-mars 2009, mis en ligne le 30 mars 2011, consulté le 30 avril 2019. URL : http://journals.openedition.org/travailemploi/4144; DOI : 10.4000/ travailemploi.4144 


\title{
À quelles conditions les salariés adhèrent-ils à un projet de crèche?
}

\author{
Yvan Barel (*), Sandrine Frémeaux (**)
}

\begin{abstract}
L'entrée massive des femmes sur le marché du travail et le développement des couples à deux carrières ont relancé l'intérêt porté aux actions menées par l'entreprise en faveur de la conciliation vie professionnelle - vie personnelle. Parce que l'incidence économique et sociale des mesures d'aide à la conciliation est incertaine, l'objet de la présente étude est de mieux comprendre les perceptions des salariés portant sur un projet d'aide à la conciliation. Une enquête qualitative et quantitative a été menée au sein d'un centre commercial français comprenant près de 650 salariés afin d'évaluer l'adhésion de ces derniers à un projet de cofinancement d'une crèche associative et d'analyser les conditions de réussite d'un service d'aide à la conciliation. Les résultats montrent qu'avant la mise en place d'un tel service, il est pertinent de favoriser le développement de pratiques managériales respectueuses des contraintes personnelles.
\end{abstract}

Les multiples bouleversements démographiques et sociaux liés à l'entrée massive des femmes sur le marché du travail, au développement des couples à deux carrières et à l'augmentation du nombre de divorces et de familles monoparentales ont accru les difficultés de conciliation vie professionnelle - vie personnelle (GARNER et al., 2004 ; SAINT-ONGE et al., 2002).

Selon l'Observatoire Thalys (2001) et l'enquête Histoire de vie/construction des identités réalisée en 2003 par l'Insee, les jeunes salariés se plaignent davantage que leurs aînés d'un débordement du travail sur la famille. Si la politique française de réduction du temps de travail qui s'est principalement traduite par la diminution légale de la semaine de travail a permis aux parents de jeunes enfants de mieux concilier leurs vies professionnelle et familiale (Fagnani, Letablier, 2003), elle a été également l'occasion pour les salariés d'exprimer de nouvelles attentes à l'égard de l'entreprise en matière de conciliation. À considérer l'enquête réalisée par le CREDOC en septembre 2005 pour la Caisse nationale des allocations familiales portant sur les aspirations des Français dans le domaine de la petite enfance, ces attentes concernent davantage les services d'accueil collectifs que les aides financières (PARIS, 2007).

De leur côté, les employeurs ne restent pas indifférents : l'enquête Familles et Employeurs menée en

\footnotetext{
* Maître de conférences HDR en sciences de gestion, Laboratoire d'économie et de management de NantesAtlantique (LEMNA) : yvan.barel@univ-nantes.fr ** Professeur associé, Audencia. Nantes école de management: sfremeaux@audencia.com
}

France en 2004-2005 par l'Ined et l'Insee auprès de 2673 établissements d'au moins 20 salariés montrent que plus des trois quarts des employeurs estiment qu'ils doivent aider les salariés à coordonner leur travail avec leur vie familiale (LEFÈvRE et al., 2008, 2007). Sensibles aux difficultés auxquelles les parents se confrontent désormais pour trouver une place en crèche, difficultés d'autant plus grandes que la crèche est devenue le mode de garde préféré des Français, les employeurs considèrent, selon l'enquête précitée, que l'offre de garde aux enfants des salariés de l'entreprise serait utile et répondrait à une forte demande de la part des salariés.

Et pourtant, ce service de crèche demeure exceptionnel. Alors qu'au début du $\mathrm{XX}^{\mathrm{e}}$ siècle, il n'était pas rare de voir en France des entreprises «paternalistes» répondre aux besoins personnels des salariés et de leurs familles, l'implication des entreprises dans le domaine spécifique de la garde des enfants est devenue une curiosité. Seules quelques entreprises privées françaises qui relèvent le plus souvent de la fonction publique hospitalière ou territoriale ont pris l'initiative d'une telle mesure: moins de $2 \%$ des employeurs français disposent d'une crèche ou de places en crèche. Les projets relatifs aux crèches restent tout aussi rares. En 2005, soit un an après l'instauration du «crédit d'impôt famille» visant à inciter les entreprises à créer ou à cofinancer une crèche, seulement $1 \%$ des établissements ne proposant pas déjà de places en crèche envisageaient de le faire, et seulement seize employeurs français ont déclaré leur offre de garde.

C'est que l'impact réel de ces initiatives est difficile à mesurer. Les dirigeants visent à promouvoir des valeurs véhiculées dans l'entreprise, à réguler 
l'absentéisme, à favoriser l'implication et la fidélisation des salariés ou à faciliter les recrutements (Eydoux et al., 2008; DAUNE-RICHARD et al., 2007). Certaines études ont bien noté ces effets, en particulier, une réduction du turnover (YoungBLOOD, Chambers-Cook, 1984; Milkovich, Gomez, 1976) ou une augmentation de la satisfaction (CONNELLY et al., 2004; Ezra, Deckman, 1996; Kossek, Nichol, 1992), mais la plupart des recherches émettent des réserves concernant l'incidence des dispositifs d'aide à la petite enfance sur la performance économique et sociale (Goff et al., 1990; Miller, 1984).

Si l'efficacité d'une création ou d'un financement de crèche est incertaine, c'est en partie en raison de la diversité des représentations des salariés. L'objet de la présente étude est précisément d'analyser les perceptions des salariés portant sur un projet de cofinancement d'une crèche associative et de suggérer des clefs de réussite pour la mise en place de ce dispositif d'aide à la conciliation. Pour ce faire, une enquête sur le thème de la conciliation des sphères professionnelle et domestique a été menée au sein d'un centre commercial français composé d'un hypermarché et de quaranteneuf boutiques marchandes, et regroupant au total près de 650 salariés. La direction du groupement d'intérêt économique (GIE) a exprimé son intérêt pour le projet interentreprises suivant: en échange d'un financement partiel, chaque entreprise pourrait obtenir une attribution prioritaire de places pour un ou plusieurs de ses salariés (à un tarif non préférentiel) dans une crèche associative à la plage horaire particulièrement étendue (de 7 heures à 21 heures). La principale motivation de la direction en faveur de ce projet était liée à un projet de communication externe: «La grande distribution souffre d'une mauvaise image. On l'accuse de tous les maux [...]. On a donc intérêt à faire parler de nous en bien, montrer qu'on a des comportements citoyens. On a tout à y gagner que ce soit auprès des pouvoirs publics, des clients ou des salariés $»$.

Le choix du terrain de recherche est pertinent dans la mesure où le conflit travail - famille paraît a priori élevé:

- la largeur des plages d'ouverture des centres commerciaux et les fortes variations de présence de la clientèle conduisent souvent le personnel à travailler tôt le matin, tard le soir, le samedi et les périodes de fêtes (BARET et al., 1998);

- la gestion des ressources humaines, plus quantitative que qualitative dans le secteur de la grande distribution, conduit à la prédominance de formes de management plutôt autoritaires, laissant peu de place à l'écoute et à l'empathie (BAREL, 2000);

- le centre commercial en question est composé d'une très large majorité de personnel féminin, en général davantage sollicité que le personnel masculin par les contraintes extraprofessionnelles (MÉDA, 2002).

\section{Quelles réponses organisation- nelles au conflit travail - famille?}

Dans la continuité des études anglo-saxonnes de Kanter (1977), Orthener et Pittman (1986) et Hall et Richter (1988), KiRChMEYER (1995) a proposé une classification des réponses organisationnelles apportées par les entreprises aux difficultés de conciliation entre les sphères professionnelle et privée: la «séparation », l' «intégration» et le «respect». L'entreprise met en œuvre le principe de séparation lorsqu'elle ne se préoccupe pas de la vie personnelle de ses salariés. Elle met en œuvre la logique de l'intégration lorsqu'elle supprime la frontière entre les vies professionnelle et personnelle en proposant aux salariés des services : services d'aide à la garde d'enfants, implantation ou financement partiel d'une crèche d'entreprise, services de lavage de voitures, pressing et repassage, conseil juridique et financier, livraison d'alimentation, etc. Le principe de respect préconisé par HALL RICHTER (1988) suppose le maintien d'une frontière entre vie professionnelle et vie privée. Il s'agit pour l'employeur d'aider les salariés à assumer leurs responsabilités en prenant en compte leurs contraintes personnelles et en leur accordant des horaires flexibles. Les critères discriminants réellement retenus par KIRCHMEYER (1995) pour caractériser les pratiques de respect sont la considération par les managers des activités extraprofessionnelles et l'autonomie laissée aux salariés dans l'organisation de leur temps de travail.

Si des recherches ont remis en cause l'efficacité du modèle de l'intégration en montrant que celui-ci n'avait pas d'incidence positive sur l'engagement organisationnel (HALL, Richter, 1988; KIRCHMEYER, 1995), elles ne se sont pas intéressées à la façon dont les salariés perçoivent cette réponse organisationnelle (McDonAld et al., 2005). Par contre, la littérature traitant des sources du conflit travail - famille est abondante. Dans la continuité des travaux de Frone et al. (1992) qui opposent deux catégories de variables explicatives du conflit travail - famille, l'une liée aux contraintes individuelles et familiales, l'autre aux contraintes professionnelles et organisationnelles, la présente étude reprend ces mêmes variables pour voir dans quelle mesure elles exercent une influence sur l'adhésion des salariés à un projet de cofinancement d'une crèche associative.

Les travaux anglo-saxons les plus récents, en particulier ceux de ANDERSON et al. (2002) et de Duxbury et Higgins (2001), ainsi que ceux issus de la littérature française de PAILHÉ et Solaz (2006), MÉdA et Orain (2002) et ThÉvenet (2001) ont souligné l'importance de certaines variables individuelles explicatives du conflit travail - famille: le genre, l'âge ainsi que l'existence, le nombre et l'âge des enfants. 


\section{Genre}

En France, les femmes assurent encore $80 \%$ du temps domestique total, et le temps consacré à ces tâches par les hommes n'aurait guère changé ces trente dernières années (PAIlhé, Solaz, 2006; Garner et al., 2005; Dumontier, Pan Ke Shon, 2002). Parce que les femmes consacrent plus de temps que les hommes aux tâches domestiques, elles ont davantage le sentiment de manquer de temps et sont plus sensibles que les hommes à un allégement des contraintes temporelles liées à la vie professionnelle (CRENNER, 1999; FERMANIAN et al., 1999).

\section{Âge}

À une certaine période de la vie, les contraintes professionnelles et familiales peuvent être plus fortes. Ce sont principalement les salariés appartenant à la classe d'âge intermédiaire qui peuvent être confrontés à de nouvelles responsabilités dans les deux sphères de vie et connaître un conflit travailfamille plus important (DUXBURY et al., 1994; GreEnhaus et al., 1987).

\section{Statut de parent, nombre et âge des enfants}

Le fait d'être parent, le jeune âge et le nombre d'enfants accroissent le conflit travail - famille (Micheaux, Monso, 2007). Au nombre d'enfants, il faut ajouter la diversité des âges des enfants : lorsque ces derniers nécessitent différents modes de prise en charge, les contraintes personnelles sont sensiblement plus élevées (DuXBury, Higgins, 2001).

Des variables professionnelles susceptibles d'avoir un impact sur le conflit travail - famille ont été également évoquées, notamment dans les études anglo-saxonnes de BrEAUgh et Frye (2008), NiKANDrou et al. (2008) et ANDERSON et al. (2002) ainsi que dans les études françaises effectuées par Alis, Dumas (2003), Fagnani, Letablier (2003) et MÉDA (2002): la prise en compte des contraintes personnelles du salarié par le supérieur hiérarchique, la prévisibilité des horaires et le temps consacré au travail.

\section{Prise en compte par le supérieur hiérarchique des contraintes personnelles du salarié}

Nombre d'études montrent l'importance du soutien apporté par le supérieur hiérarchique, qui peut se traduire par une considération des aspirations personnelles dans l'organisation des congés et des réunions, une acceptation des horaires variables ou une tolérance envers certains appels téléphoniques personnels (THÉVENET, 2001; GUÉRIN et al., 1997). Cette souplesse de fonctionnement favorise notamment l'écoute et l'assistance lorsque les salariés sont confrontés à des situations de crise (WARREN,
Johnson, 1995; Goff et al., 1990). Si le soutien apporté par le supérieur hiérarchique diminue le conflit travail - famille (BreAugh, FrYe, 2008; NiKANDROU et al., 2008; ANDERSON et al., 2002; Macmanus et al., 2002; Thomas, Ganster, 1995 ; WARREN, JohNSON, 1995), l'absence de considération des contraintes personnelles peut au contraire accroître un tel conflit.

\section{Prévisibilité des horaires}

La souplesse des horaires de travail et la coordination des emplois du temps des salariés et de leurs proches sont de nature à faciliter la conciliation entre la vie professionnelle et la vie personnelle (Greenhaus, Beutell, 1985). À l'inverse, l'imprévisibilité des horaires constitue une source de conflit (VoyAnDOFF, 1989).

\section{Temps consacré au travail}

Selon Kossek (1990), les salariés travaillant à temps plein sont confrontés à des difficultés de conciliation plus élevées que les salariés à temps partiel. Au contraire, BARTOLOMÉ et Evans (1980) montrent le rôle secondaire joué par le temps de travail effectif. L'analyse du nombre d'heures effectuées ne serait pas suffisante. Elle doit s'accompagner d'une analyse du jugement porté par les salariés sur le temps consacré à la sphère professionnelle (Alis, Dumas, 2003; MÉda, 2002; Brunhes et al., 2001). Ceux qui considèrent accorder trop de temps au travail vivent un conflit travail-famille plus élevé.

La question se pose de savoir s'il existe un lien entre les sources de conflit travail-famille et les perceptions des salariés concernant le projet de cofinancement d'une crèche associative. Les salariés confrontés à un conflit travail-famille élevé seraient-ils davantage enclins à percevoir de manière positive une aide relevant du modèle de l'intégration? Autrement dit, l'adhésion des salariés au projet est-elle liée aux contraintes individuelles et professionnelles précitées?

Pour répondre à ces questions, nous avons testé le modèle schématisé dans la figure 1 qui met en évidence la formulation des deux hypothèses de recherche suivantes:

-H1 : l'adhésion au projet d'aide à la conciliation est liée aux caractéristiques personnelles et familiales suivantes : genre féminin (a), classe d'âge intermédiaire (b), statut de parent (c), présence d'au moins un enfant de moins de trois ans (d);

$-\mathrm{H} 2$ : l'adhésion au projet d'aide à la conciliation est liée aux caractéristiques professionnelles et organisationnelles suivantes: absence de prise en compte des contraintes personnelles par le supérieur hiérarchique (a), imprévisibilité des horaires (b), temps de travail important (c), jugement d'un temps trop important consacré au travail (d). 
Figure 1: Modèle de la recherche

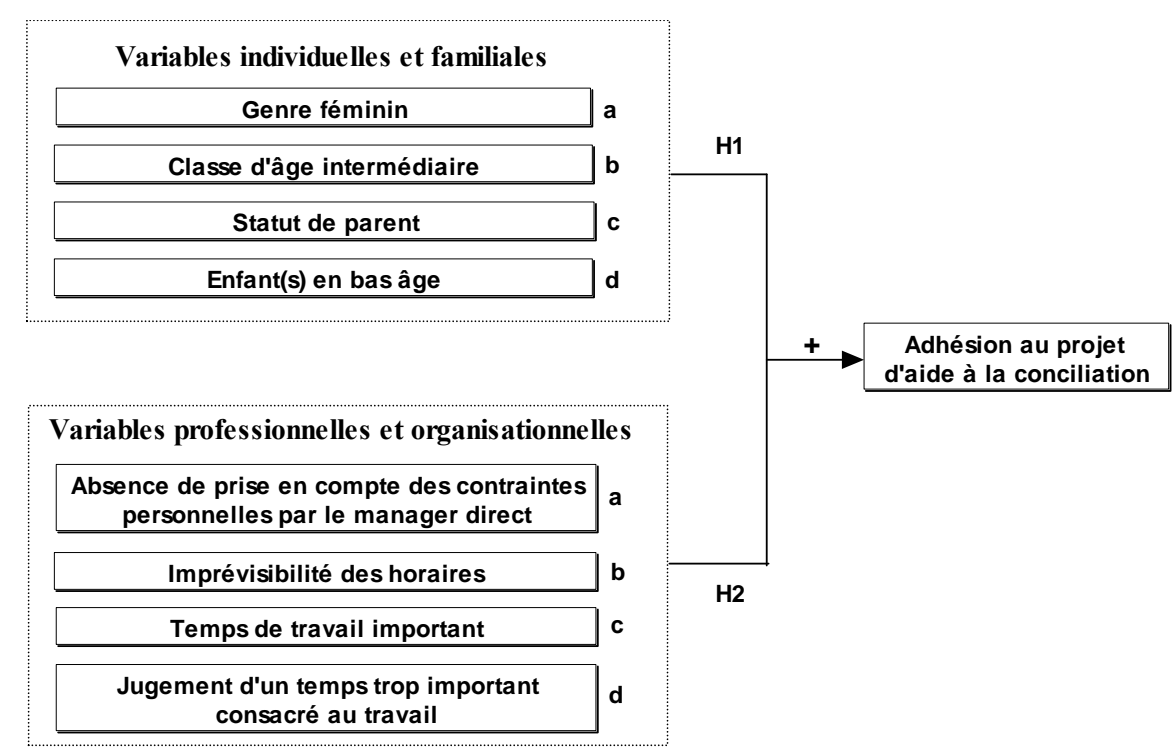

\section{Encadré 1 \\ Méthodologie}

- Modes de recueil de l'information et échantillons

La période d'investigation s'est décomposée en deux phases:

- Une phase qualitative durant laquelle des entretiens semi-directifs d'une durée d'environ une heure et dix minutes ont été menés dans des lieux neutres (cafés, box de négociation, salles de réunion) auprès d'un échantillon transversal et représentatif de 36 salariés du centre commercial (cf. tableau 1).

Tableau 1: Échantillon interrogé lors des entretiens semi-directifs

\begin{tabular}{|l|l|l|l|l|}
\hline \multicolumn{1}{|c|}{ Genre } & \multicolumn{1}{|c|}{ Âge } & \multicolumn{1}{c|}{ Enfants } & \multicolumn{1}{c|}{ Statut professionnel } & \multicolumn{1}{c|}{$\begin{array}{c}\text { Situation } \\
\text { maritale }\end{array}$} \\
\hline $\begin{array}{l}\text { Femmes }: 22 \\
\text { Hommes }: 14\end{array}$ & $\begin{array}{l}\text { Moyenne }: 34 \text { ans } \\
\text { Moins de } 40 \text { ans }: 24 \\
\text { Plus de } 40 \text { ans }: 12\end{array}$ & $\begin{array}{l}\text { Sans enfants }: 10 \\
\text { Avec enfants }: 26\end{array}$ & $\begin{array}{l}\text { Cadres : } 10 \\
\text { Non-cadres }: 26\end{array}$ & $\begin{array}{l}\text { Célibataires : } 6 \\
\text { Couples : } 30\end{array}$ \\
\hline
\end{tabular}

- Une phase quantitative durant laquelle un questionnaire a été administré auprès des 650 salariés du centre commercial. Les entretiens précédemment réalisés ont contribué à son élaboration ainsi qu'à l'interprétation des résultats statistiques des questionnaires retournés, à savoir 311 dont 300 exploitables (soit un taux de réponse de $46 \%$ ). $78 \%$ des répondants sont des femmes; $69 \%$ ont moins de 36 ans; $74 \%$ vivent en couple (mariés ou non); ils ont en moyenne 1,21 enfant (51\% n'en ont aucun; $13 \%$ en ont un; $22 \%$ en ont deux; $14 \%$ en ont trois ou plus). Parmi ceux qui ont des enfants ( $49 \%$ de l'échantillon), près des deux tiers ont au moins un enfant dont l'âge est inférieur ou égal à trois ans. $92 \%$ des salariés de l'échantillon disposent d'un contrat à durée indéterminée et $39 \%$ d'un contrat à temps partiel.

- Mesures des variables utilisées dans le questionnaire

- Mesure de l'adhésion au projet

Les salariés devaient répondre sur une échelle composée de quatre items ( 1 non; 2 plutôt non; 3 plutôt oui; 4 oui) à la question de savoir s'ils étaient favorables au projet d'attribution de places dans une crèche cofinancée par les entreprises. Une valeur moyenne faible (minimum égal à 1) correspond à un rejet du projet, tandis qu'une valeur élevée (maximum égal à 4) traduit une forte adhésion. La moyenne théorique s'élève à 2,5 et signifie une opinion neutre.

\section{- Mesure des variables personnelles et professionnelles}

Les variables personnelles sont le genre (masculin/féminin), l'âge (moins de 25 ans/25-35 ans/36-45 ans/ plus de 45 ans), le statut de parent (oui/non) et le fait d'avoir au moins un enfant de moins de trois ans (oui/ non). Les variables professionnelles sont la prise en compte des contraintes personnelles par le supérieur hiérarchique (mesurée par l'échelle: jamais/quelquefois/souvent/toujours), la prévisibilité des horaires (même échelle de mesure), le temps de travail (temps complet/temps partiel) et le sentiment de consacrer trop de temps au travail (oui/non). 


\section{Variables individuelles et familiales et perception du projet d'aide à la conciliation}

Globalement, les salariés se déclarent plutôt favorables au projet de cofinancement de la crèche (moyenne égale à 2,94) ( $c f$. tableau 2). Si le genre s'avère être une variable peu significative, la tendance va malgré tout dans le sens de l'hypothèse précitée, les femmes ayant en moyenne une opinion plus positive du projet de cofinancement de la crèche (H1a partiellement validée). Les salariés relevant de la classe d'âge intermédiaire expriment une plus forte adhésion que les salariés des classes extrêmes, mais la différence n'est pas significative (H1b non validée). En revanche, la présence d'au moins un enfant en bas âge (de moins de 3 ans) favorise la perception positive du projet (H1d validée). La diversité des âges des enfants accroît également le besoin d'être aidé et l'adhésion à la mesure: «Depuis l'arrivée au monde de notre petite dernière, mon mari et moi, on a besoin d'être deux pour accompagner et aller chercher les enfants. Mon mari s'occupe des trajets à l'école pour les deux grands, moi je m'occupe d'aller à la crèche pour le bébé. Il arrive aussi qu'un seul parent doive s'occuper des trois enfants. Et alors là, c'est du sport. C'est sûr que ça serait plus pratique une crèche ouverte de 7 heures à 21 heures comme c'est prévu dans le projet. Sinon avec mes horaires particuliers, on est coincé...» (Vendeuse). Certains salariés ayant des enfants plus grands n'ont pas accueilli favorablement le projet dans la mesure où ils ne projettent pas d'avoir d'autres enfants et n'ont pas bénéficié par le passé d'une aide dispensée par l'entreprise: «Une crèche... et puis quoi encore? Des terrains de tennis à la disposition des salariés sur le parking des clients? Moi j'ai quatre enfants, le plus jeune va passer son bac cette année. Je me suis toujours débrouillée par mes propres moyens. Pourquoi ça devrait être plus facile pour les jeunes?» (Vendeuse). Dans ce cas, c'est moins l'absence de contraintes familiales ou professionnelles que l'impossibilité de tirer parti de la mesure qui explique le refus du projet. De même que les salariés qui ne bénéficient pas de la mesure en ont une perception plus négative (GROVER, 1991), les salariés qui ne projettent pas d'en bénéficier semblent en avoir une vision moins positive. Aussi, il n'est guère surprenant que les salariés parents et non parents partagent globalement la même opinion (H1c non validée). La population des salariés parents regroupe des parents d'enfants de moins de trois ans très favorables au projet et des parents d'enfants plus âgés dont l'adhésion est moindre. Dans la population des salariés non parents, l'opinion favorable de ceux qui pensent avoir des enfants contrebalance la perception plutôt négative de ceux qui ne projettent pas d'en avoir. Recouvrant des situations diversifiées, le statut de parent n'est donc pas un critère discriminant.

\section{Variables professionnelles et organisationnelles et perception du projet d'aide à la conciliation}

Le sentiment de consacrer trop de temps à son travail n'a pas d'incidence sur la perception du projet (H2d non validée) ( $c f$. tableau 2). Et contrairement à l'hypothèse $2 \mathrm{c}$, ce sont les salariés travaillant à temps partiel qui sont les plus favorables au projet. Ce résultat peut paraître contre intuitif dans la mesure où ces salariés vivent $a$ priori un moindre conflit travail - famille. Les entretiens laissent entrevoir trois raisons explicatives:

- les salariés travaillant à temps partiel ont des horaires atypiques et incertains qui créent un besoin de soutien fiable: «À deux heures près, je ne sais jamais vraiment à quelle heure je quitte ma caisse. $\mathrm{Y}$ a toujours des remplacements de dernière minute. Ce qui est bien avec une crèche avec grande amplitude horaire, c'est qu'on peut travailler l'esprit tranquille» (Hôtesse de caisse).

- des salariés ont choisi le temps partiel précisément parce qu'ils sont confrontés à de fortes contraintes familiales: «Je travaille 20 heures par semaine mais je dois m'occuper de mon bébé et de ma mère âgée qui est malade. Je suis sur les rotules. C'est sûr qu'une place en crèche, ça faciliterait la vie. C'est tellement difficile d'avoir une place». (Vendeuse).

- le sentiment d'avoir déjà obtenu de leur employeur des concessions leur permettant de mieux vivre la conciliation entre les deux sphères favorise une attitude positive de ces salariés: «J'ai la chance d'avoir un temps partiel qui me convient. Certains de mes collègues n'ont pas cette chance. Je comprends qu'ils rejettent l'idée de la crèche. Pour moi, tout ce qui va dans le sens de l'amélioration des conditions de travail est une bonne chose» (Vendeur).

Conformément à l'hypothèse $2 \mathrm{~b}$, les salariés ayant des horaires imprévisibles sont plus favorables au projet de cofinancement d'une crèche, probablement parce que l'incertitude des horaires de travail renforce le conflit travail - famille et donc le besoin de bénéficier d'un soutien concret.

Par contre, les salariés qui se plaignent d'un manque de considération de leurs situations personnelles par le management direct sont de manière très significative moins favorables au projet d'aide (H2a non validée) : ils ont tendance à trouver le projet superflu et incohérent, en tant qu'il ne règle pas les difficultés quotidiennes en grande partie liées à l'organisation rigide du travail. Les problèmes les plus fréquents évoqués par les salariés concernent les réunions fixées à des heures tardives par le supérieur hiérarchique, l'absence d'horaires variables et l'impossibilité de faire moins d'heures ou de prendre des jours de congés le mercredi : "J'ai demandé à mon chef si je pouvais faire moins d'heures le mercredi à cause 
Tableau 2: Adhésion au projet de cofinancement d'une crèche et variables individuelles et organisationnelles

\begin{tabular}{|c|c|c|c|c|}
\hline & Modalités & Effectifs & \begin{tabular}{|c|} 
Adhésion au projet de cofi- \\
nancement d'une crèche \\
1 non; 2 plutôt non; \\
3 plutôt oui ; 4 oui
\end{tabular} & $\begin{array}{l}\text { Test de } \\
\text { Fisher }\end{array}$ \\
\hline \multicolumn{5}{|l|}{ Variables individuelles } \\
\hline \multirow{2}{*}{ Genre } & Masculin & 64 & 2,89 & \multirow{2}{*}{$(*)$} \\
\hline & Féminin & 223 & 2,96 & \\
\hline \multirow{4}{*}{ Âge } & Moins de 25 ans & 84 & 2,91 & \multirow{4}{*}{ NS } \\
\hline & $25-35$ ans & 116 & 2,98 & \\
\hline & $36-45$ ans & 68 & 2,92 & \\
\hline & Plus de 45 ans & 20 & 2,87 & \\
\hline \multirow{2}{*}{ Au moins un enfant } & Oui & 140 & 2,96 & \multirow{2}{*}{ NS } \\
\hline & Non & 144 & 2,93 & \\
\hline \multirow{2}{*}{ Au moins un enfant de moins de 3 ans } & Oui & 92 & 3,14 & \multirow{2}{*}{$(* *)$} \\
\hline & Non & 196 & 2,86 & \\
\hline \multicolumn{5}{|l|}{ Variables organisationnelles } \\
\hline \multirow{2}{*}{ Prise en compte des contraintes personnelles } & Jamais ou quelquefois & 148 & 2,59 & \multirow{2}{*}{$(* * *)$} \\
\hline & Souvent ou toujours & 152 & 3,29 & \\
\hline \multirow{2}{*}{ Horaires prévisibles } & Jamais ou quelquefois & 92 & 3,11 & \multirow{2}{*}{$(* *)$} \\
\hline & Souvent ou toujours & 200 & 2,86 & \\
\hline \multirow{2}{*}{ Temps de travail } & Temps complet & 173 & 2,79 & \multirow{2}{*}{$(* *)$} \\
\hline & Temps partiel & 111 & 3,17 & \\
\hline \multirow{2}{*}{ Sentiment de consacrer trop de temps au travail } & Oui & 64 & 2,95 & \multirow{2}{*}{ NS } \\
\hline & Non & 236 & 2,94 & \\
\hline
\end{tabular}

Note de lecture: les seuils de significativité sont respectivement égaux à $1 \%(* * *), 5 \%(* *)$ et $10 \%(*)$.

$\mathrm{NS}=$ non significatif.

Les modalités «Jamais» et «Quelquefois» ainsi que les modalités «Souvent» et «Toujours» ont été regroupées afin d'augmenter la significativité des résultats.

de mes enfants et de compenser sur les autres jours de la semaine. Certains de mes collègues étaient prêts à travailler plus le mercredi pour m'arranger. Mais le chef a refusé de manière catégorique en disant que si on commence à s'occuper de la vie personnelle des uns et des autres on ne s'en sort plus. Et voilà que maintenant j'apprends qu'on veut financer une crèche associative. C'est quoi la logique?» (Employée libre-service). De plus, les employés acceptent difficilement d'avoir à dépasser le nombre d'heures hebdomadaires prévu au contrat, alors que ces heures ne sont quasiment jamais payées ou récupérées: «Si leur souci est véritablement de nous aider à concilier notre vie professionnelle avec notre vie privée, alors ils n'ont qu'à commencer par nous payer les heures sup !» (Vendeuse). Par ailleurs, les cadres intermédiaires (chefs de rayon ou seconds de rayon) qui voulaient réduire leur temps de présence dans l'hypermarché en ramenant du travail à leur domicile et qui en ont été dissuadés par l'attitude de leurs supérieurs hiérarchiques remettent en cause l'organisation du travail: «J'aimerais bien que le directeur et les chefs de secteur ne s'obsèdent pas sur notre temps de travail. Si t'arrives pas avant 8 heures et si tu repars pas après 18 heures 30 , six jours sur sept, t'es mal vu. Y a du boulot que je pourrais tout aussi bien faire chez moi, et même mieux. J'ai l'impression de ne pas voir grandir mes enfants. Je ne peux ni accompagner le plus petit à la crèche ni aller le chercher. Le mercredi, rebelote pour leurs activités sportives, c'est ma femme qui s'occupe de tout» (Chef de rayon). L'importance accordée au temps de présence des managers est ainsi particulièrement mal vécue par les jeunes diplômés qui estiment un contrôle par les résultats suffisant: «On a des objectifs très précis et ambitieux à atteindre. Qu'on nous laisse au moins libres de la façon d'organiser notre temps de travail» (Jeune chef de rayon). Entrevoyant le projet comme un signe de méconnaissance des besoins réels, les salariés, cadres et non cadres, craignent un renforcement des exigences du supérieur hiérarchique en matière de disponibilité : «Ma femme ne pense pas que mon chef me laissera quitter mon boulot à la même heure si notre bébé était accepté dans la crèche financée par l'entreprise avec ses horaires à rallonge. Elle a peut-être bien raison » (Chef de rayon adjoint). Ces résultats montrent que les salariés considèrent comme prioritaire la reconnaissance d'une plus grande autonomie dans l'organisation de leur temps de travail. 
Dès lors que l'entreprise projette de créer ou de cofinancer une crèche, une variable relative à la situation personnelle des salariés mérite une attention préalable: la présence d'un ou plusieurs enfants de moins de trois ans. Le fait d'avoir des enfants en bas âge semble accroître sensiblement la perception positive d'une mesure relevant de la logique d'intégration. Ce constat confirme les études qui avaient d'ores et déjà révélé que les projets de création ou de financement de crèches connaissent un succès dans les entreprises comprenant un grand nombre de parents de jeunes enfants (KonRad, Mangel, 2000).

Cette analyse des caractéristiques sociodémographiques des salariés qui s'accompagne souvent de celle des structures d'accueil environnantes, du coût et des avantages fiscaux de la mesure projetée, ne semble pas être suffisante. Un programme de conciliation suppose également une analyse des pratiques managériales. L'adhésion au projet est étroitement subordonnée à la considération par le management direct des contraintes personnelles des salariés. Bien que réduisant le conflit travail - famille, le respect des situations personnelles augmente les chances d'adhésion au projet. Ce résultat s'inscrit dans la continuité de l'approche développée par THÉvENET (2001) selon laquelle il est préférable, avant de créer de nouveaux services, de remettre à plat le dispositif managérial existant. Il permet aussi de comprendre pourquoi un programme d'aide à la conciliation a plus de chances de réussir dans les établissements qui ont récemment mis en place des changements organisationnels (LEFÈvRE et al., 2007). Il confirme enfin l'un des principaux résultats de l'enquête précitée Familles et Employeurs menée en France en 2004-2005: la souplesse et l'aménagement des horaires en fonction des contraintes personnelles doivent devenir un élément déterminant de la conciliation vie professionnelle - vie personnelle (LEFÈVRE et al., 2008).

Notre étude montre que l'importance des responsabilités domestiques auxquelles les salariés sont confrontés ne garantit pas la réussite d'un projet d'aide à la conciliation vie professionnelle - vie personnelle. Dans un contexte organisationnel où les managers ne prennent pas toujours en compte les situations personnelles, il semble préférable d'éviter la mise en œuvre d'un projet relevant de la logique d'intégration. Dès lors qu'une offre de garde des jeunes enfants du personnel serait souvent en décalage avec les pratiques managériales existantes, on comprend mieux le constat effectué par LEFÈVRE et al. (2007) selon lequel la plupart des entreprises françaises renoncent, en dépit des incitations légales, à la mise en œuvre d'un tel projet.

Dans les études empiriques futures, une démarche similaire pourrait être entreprise pour savoir si les mêmes résultats seraient obtenus à l'occasion d'autres projets d'aide à la conciliation qui relèveraient du modèle de l'intégration: création d'une crèche d'entreprise, services de garde d'enfants à domicile, services de courses, services d'aide aux personnes âgées, etc.

Parce que l'octroi de services personnels aux salariés suscite bien des peurs et fantasmes, il est important que l'entreprise ne tienne pas pour acquises les retombées positives de toute démarche favorable à la conciliation des vies professionnelle et personnelle. C'est à la condition que des pratiques de respect soient mises en place dans l'ensemble des services de l'entreprise qu'il peut s'avérer opportun d'aller plus loin pour se rapprocher du modèle de l'intégration et satisfaire les besoins particuliers de certains salariés. 


\section{Bibliographie}

Alis D., Dumas M. (2003), «35 heures, soutien organisationnel perçu et harmonisation vie familiale/vie professionnelle», Revue de gestion des ressources humaines, $\mathrm{n}^{\circ} 50$, octobre-novembre-décembre, pp. 37-56.

Anderson S.E., Coffey B.S., Byerly R.T. (2002), "Formal organizational initiatives and informal workplace practices: links to work-family conflict and job-related outcomes", Journal of Management, Vol. 28, Issue 6, pp. 787-810.

BAREL Y. (2000), Les interactions entre la stratégie, le manager et son équipe, Paris, L'Harmattan.

Baret C., Gadret J., Gallouj C. (1998), «Le temps de travail dans la grande distribution en France, Allemagne, Grande-Bretagne», Travail et Emploi, n ${ }^{\circ}$ 74, pp. 21-35, Dares.

Bartolomé F., Evans P.A.L. (1980), "Must success cost so much?", Harvard Business Review, mars-avril, Vol. $58, \mathrm{n}^{\circ} 2$, pp. $137-148$.

Breaugh J., Frye N. (2008), "Work-Family Conflict: The importance of Family-Friendly employment Practices and Family-Supportive Supervisors", Journal of business and Psychology, Vol. 22, Issue 4, pp. 345-353.

Brunhes, B., Clerc D., Meda D., Perret B. (2001), 35 heures: le temps du bilan, Paris, Desclée de Brouwer.

Connelli R., Degraff D.A. Willis, R. (2004), "The value of employer-Sponsored Child Care to Employees", Industrial Relations, Vol. 43, $\mathrm{n}^{\circ}$ 4, pp. 759-792.

Crenner E. (1999), «Les opinions des salarié sur la réduction de leur temps de travail», Économie et Statistique, $\mathrm{n}^{\circ} 321$ et $322,1 / 2$, Insee, pp. 149-159.

Daune-Richard A.-M., Odena S., Petrella F. (2007), «Entreprises et modes d'accueil de la petite enfance. Innovation et diversification", Dossier d'étude, $\mathrm{n}^{\circ}$ 91, CNAF.

Dumontier F., Pan Ke Shon J.-L. (2002), «L'évolution des temps sociaux au travers des enquêtes Emploi du temps», Économie et statistique, $\mathrm{n}^{\circ} 352-353$, Insee, pp. 3-13.

Duxbury L., Higgins C. (2001), Work-Life Balance In The New Millennium: Where Are We? Where Do We Need To Go?, Ottawa, Santé Canada.

Duxbury L., Higgins C., Lee, C. (1994), "Work-family conflict: a comparison by gender, family type and perceived control", Journal of family Issues, Vol. 15, pp. 449-466.

Eydoux A., Gomel B., Letablier M.-T. (2008), «Activités sociales et aménagements temporels, L'action des entreprises en faveur de la conciliation travail - vie familiale de leurs salariés», Recherches et prévisions, n 92 , pp. 9-20.
Ezra M., Deckman M. (1996), "Balancing work and family responsibilities: flextime and child care in the Federal Government", Public Administration Review, mars-avril, Vol. 56, No 2, pp. 174-179.

FAgnAni J., Letablier M.-T. (2003), «La réduction du temps de travail a-t-elle amélioré la vie quotidienne des parents de jeunes enfants », Premières synthèses, $\mathrm{n}^{\circ} 01.2$ janvier, Dares.

Fermanian J.-D., Galtier B., Lagarde S. (1999), «Réduction collective ou individuelle du temps de travail: que souhaitent les salariés?», Économie et Statistique, $\mathrm{n}^{\circ} 321$ et 322 , Insee.

Frone M.R., Russel M., Cooper M.L. (1992), “Antecedents and outcomes of work - family conflict: testing a model of the work - family interface", Journal of applied psychology, Vol. 77, pp. 65-78.

Garner H., Méda D., SÉnik C. (2004), «La difficile conciliation entre vie professionnelle et vie familiale», Premières Synthèses, $\mathrm{n}^{\circ}$ 50.3, Dares.

GarnerH., Méda D., SÉniK C. (2005), «Conciliation entre vie professionnelle et vie familiale, les leçons des enquêtes auprès des ménagers", Travail et Emploi, $\mathrm{n}^{\circ}$ 102, avril-juin, Dares, pp.57-67.

Goff S.J., Mount M.K., Jamison R.L. (1990), "Employer supported child care, work/family conflict, and absenteeism: a field study", Personnel psychology, Vol. 43, pp. 793-809.

Greenhaus J.H., Bedeian A.G., Mossholder K.W. (1987), "Work experiences and job performance and feelings of personal and family well-being", Journal of vocational behavior, Vol. 31, pp. 200-215.

Greenhaus J.H., Beutell N.J. (1985), "Sources of conflict between work and family roles", Academy of Management Journal, Vol. 10, pp. 76-88.

Grover S.L. (1991), "Predicting the perceived fairness of parental leave policies", Journal of Applied Psychology, 76(2), pp. 247-255.

Guérin G., Saint-Onge S., Haines V., Trottier R., SIMARD M. (1997), «Les pratiques d'aide à l'équilibre emploi-famille dans les organisations du Québec», Relations industrielles, vol. 52, $\mathrm{n}^{\circ}$ 2, pp. 274-303.

HAll D.T., Richter J. (1988), "Balancing work life and home life: What can organizations do to help?", Academy of Management Executive, Vol. 2, pp. 213-223.

KANTER R.M. (1977), Work and family in the United States: a critical review and agenda for research and policy, New York, Russel Sage Foundation.

KirChMEYeR C. (1995), "Managing the work-nonwork boudary: an assessment of organizational responses", Human Relations, Vol. 48, No 5, pp. 515-536. 
KonRad A.M., Mangel R. (2000), "The impact of worklife programs on firm productivity", Strategic Management Journal, Vol. 21, pp. 1225-1237.

Kossek E.E. (1990), "Diversity in child care assistance needs: employee problems, preferences, and workrelated outcomes", Personnel psychology, Vol. 43, No 4, pp. 769-791.

Kossek E.E., Nichol V. (1992), "The effects of on-site child care on employee attitudes and performance", Personnel psychology, Vol. 45, pp. 485-509.

LefÈvre C., Pailhé A., Solaz A. (2007), «Comment les employeurs aident-ils leurs salariés à concilier travail et famille?», Population et Sociétés, $\mathrm{n}^{\circ} 440$, décembre, Ined.

Lefèvre C., Pailhé A., Solaz A. (2008), «Les employeurs, un autre acteur de la politique familiale? Un état des lieux de leur participation dans la conciliation vie familiale-vie professionnelle», Recherches et Prévisions, $\mathrm{n}^{\circ}$ 92, pp. 21-31.

Macmanus K., Korabik K., Rosin H.M., Kevin KelloWAY E. (2002), "Employed mothers and the work-family interface: does family structure matter?", Human relations, Vol. 55, No 11, pp. 1295-1324.

McDonald P., Guthrie D., Bradley L., ShakespeareFINCH J. (2005), "Investigating work - family policy aims and employee experiences", Employee Relations, Vol. 27, issue 5, pp. 478-494.

MÉDa D. (2002), Le Temps des femmes. Pour un nouveau partage des rôles, Paris, Flammarion, réédition collection «Champs-Flammarion».

MÉDA D., Orain R. (2002), «Transformations du travail et de la famille: le jugement des salariés sur la réduction du temps de travail», Travail et emploi, $\mathrm{n}^{\circ}$ 90, avril, Dares, pp. 23-38.

Micheaux S., Monso O. (2007), «Faire garder ses enfants pendant son temps de travail», Insee Première, $n^{\circ} 1132$, avril, Insee.

Milkovich G.T., Gomez L.R., (1976), "Day care and selected employee work behaviors", Academy of management journal, Vol. 19, pp. 111-115.

Miller T.I. (1984), “The effects of employer-supported child care on employee absenteeism, turnover, produc- tivity, recruitment, or job satisfaction: what is claimed and what is known" Personnel psychology, Vol. 37, pp. 212-226.

Nikandrou I., Panayotopoulou L., Apopori E. (2008), "The impact of individual and organizational charactéristics on work-family conflict and career outcome", Journal of Managerial Psychology, Vol. 23, Issue 5, pp. 576-598.

Observatoire Thalys (2001), «Le temps au cœur des préoccupations des sociétés européennes», Sondage IPSOS, Paris.

Orthener D.K., Pittman J.F. (1986), "Family contributions to work commitment", Journal of Marriage and the Family, Vol. 48, pp. 573-581.

Pailhé A., Solaz A. (2006), «Vie professionnelle et naissance: la charge de la conciliation repose essentiellement sur les femmes », Population et Sociétés, $n^{\circ} 426$, Ined.

PARIs H. (2007), «Les attentes des Français vis-à-vis des modes de garde pour les jeunes enfants », Recherches et Prévisions, $\mathrm{n}^{\circ}$ 87, pp. 83-88.

Saint-Onge S., Renaud S., Guérin G., Caussignad E. (2002), «Vérification d'un modèle structurel à l'égard du conflit travail - famille», Relations Industrielles, vol. 57, $\mathrm{n}^{\circ} 3$, pp. 491-516.

ThéVenet M. (2001), «Vie professionnelle, vie privée et développement personnel», Revue française de gestion, juin-juillet-août, $\mathrm{n}^{\circ}$ 134, pp. 106-119.

Thomas L.T., Ganster D.C. (1995), "Impact of familysupportive work variables on work-family conflict and strain: a control perspective", Journal of applied psychology, Vol. 80, pp. 6-15.

Voyandoff P. (1989), "Work and family: a review and expanded conceptualization”, Journal of Social Behavior and Personality, Vol. 3, pp. 1-22.

WARREN J.A., Johnson P.J. (1995), “The impact of workplace support on work-family role strain", Family relations, Vol. 44, pp. 163-169.

Youngblood S.A., Chambers-Cook K. (1984), "Child care assistance can improve employee attitudes and behavior", Personnel Administrator, Vol. 29, pp. 45-47. 